Wright State University

CORE Scholar

Physics Faculty Publications

Physics

5-1-2009

\title{
Strain Waves, Earthquakes, Slow Earthquakes, and Afterslip in the Framework of the Frenkel-Kontorova Model
}

\author{
Naum I. Gershenzon \\ Wright State University - Main Campus, naum.gershenzon@wright.edu \\ V. G. Bykov \\ Gust Bambakidis \\ Wright State University - Main Campus, gust.bambakidis@wright.edu
}

Follow this and additional works at: https://corescholar.libraries.wright.edu/physics

Part of the Earth Sciences Commons, Environmental Sciences Commons, and the Physics Commons

\section{Repository Citation}

Gershenzon, N. I., Bykov, V. G., \& Bambakidis, G. (2009). Strain Waves, Earthquakes, Slow Earthquakes, and Afterslip in the Framework of the Frenkel-Kontorova Model. Physical Review E, 79 (5), 56601. https://corescholar.libraries.wright.edu/physics/184

This Article is brought to you for free and open access by the Physics at CORE Scholar. It has been accepted for inclusion in Physics Faculty Publications by an authorized administrator of CORE Scholar. For more information, please contact library-corescholar@wright.edu. 


\title{
Strain waves, earthquakes, slow earthquakes, and afterslip in the framework of the Frenkel-Kontorova model
}

\author{
N. I. Gershenzon, ${ }^{1,2}$ V. G. Bykov, ${ }^{3}$ and G. Bambakidis ${ }^{1}$ \\ ${ }^{1}$ Physics Department, Wright State University, Dayton, Ohio, 45435 USA \\ ${ }^{2}$ Department of Earth and Environmental Sciences, Wright State University, Dayton, Ohio, USA \\ ${ }^{3}$ Institute of Tectonics and Geophysics, Far Eastern Branch of the Russian Academy of Sciences, Khabarovsk, Russia
}

(Received 14 August 2008; revised manuscript received 7 November 2008; published 6 May 2009)

\begin{abstract}
The one-dimensional Frenkel-Kontorova (FK) model, well known from the theory of dislocations in crystal materials, is applied to the simulation of the process of nonelastic stress propagation along transform faults. Dynamic parameters of plate boundary earthquakes as well as slow earthquakes and afterslip are quantitatively described, including propagation velocity along the strike, plate boundary velocity during and after the strike, stress drop, displacement, extent of the rupture zone, and spatiotemporal distribution of stress and strain. The three fundamental speeds of plate movement, earthquake migration, and seismic waves are shown to be connected in framework of the continuum FK model. The magnitude of the strain wave velocity is a strong (almost exponential) function of accumulated stress or strain. It changes from a few $\mathrm{km} / \mathrm{s}$ during earthquakes to a few dozen $\mathrm{km}$ per day, month, or year during afterslip and interearthquake periods. Results of the earthquake parameter calculation based on real data are in reasonable agreement with measured values. The distributions of aftershocks in this model are consistent with the Omori law for temporal distribution and a $1 / r$ for the spatial distributions.
\end{abstract}

DOI: 10.1103/PhysRevE.79.056601

PACS number(s): 05.45.Yv, 91.10.Kg

\section{INTRODUCTION}

According to modern plate tectonics, the earth crust movement is caused by mantle convection, whereby faults on the earth's surface arise along the lines of highest horizontal gradient of the flow velocity. The comparatively rigid plates move slowly relative to each other along the faults at a rate of a few centimeters per year. These movements are essentially nonuniform in time and space and accompanied by the phenomena of earthquakes, postseismic relaxation and creep, and seismic gaps [1]. The origins of crustal deformation (strain or stress waves) and earthquake migration along active faults are current problems in plate dynamics. Since a strain wave implies a stress wave and vice versa, we shall discuss only the strain wave in what follows.

Migration of large earthquakes has been explained in terms of progressive failure caused by stress redistribution along faults. Each seismic slip increases stress in the neighboring fault segment, thus facilitating nucleation of the ensuing large event. An alternative explanation is that the regular seismic migration is driven by strain propagation (deformation fronts or strain waves), which adds tectonic stress and, hence, triggers large earthquakes in critically stressed fault segments.

Since there is no explicit experimental observations of strain wave the question arises if such phenomena do exist? The problem of slow strain waves has been discussed in the literature for over 30 years. The existence of these waves was theoretically grounded and inferred implicitly from geophysical responses but explicit experimental evidence has been far insufficient. The difficulty in detection of strain waves in the earth is due to the absence of a specific type of detector capable of recording these waves and also in the ambiguity of interpretation of the observed values and the absence of an adequate theory which could enable one to determine the parameters of these waves and the most probable location for recording them.

In terms of wave parameters, strain waves are similar to seismic waves but have ultralow propagation velocities $V$ $=O(10 \mathrm{~km} / \mathrm{yr})$, predominant ultralow frequencies $f$ $=O\left(10^{-7} \mathrm{~Hz}\right)$, and very long wavelength $\lambda=O(10 \mathrm{~km})$. These peculiarities make instrumental observations of the strain wave propagation effect extremely difficult. We think these are such waves which are responsible for slow redistribution of stresses within the lithosphere.

Presently the most feasible way of strain wave detection is recording of perturbation migration in the geophysical fields. A material carrier of such perturbations is necessary, and this can be the wave only, as geomass movement does not occur. A wave mechanism appears to be more realistic than any other probable mechanism.

Our motivation for this study was to construct a mathematical model (in point of fact, a simple heuristic model) which describes the mechanism generating strain waves along faults and the observable effects associated with them. The spasmodic local motion along a fault requires essentially less external stress than spatially and temporally uniform motion. This process is analogous to plastic deformation in crystal materials. The plasticity is realized by the movement of edge dislocations, which are a certain type of crystal lattice defect. Such movement requires much less stress than is necessary for uniform relative displacement of crystal parts along a crystal plane [2]. So the movement of an edge dislocation in a crystal slip plane has some common features with a strain wave propagating along a transform fault.

Here the processes of nucleation, movement, and interaction of edge dislocations are described by the FrenkelKontorova (FK) model [3], this is simplified single fault model. In the continuum limit the FK model is described by the sine-Gordon (SG) equation [4-6]. Solutions of the SG 
equation can be used to model various phenomena of plate movement such as strain waves, regular earthquakes, slow earthquakes, and afterslip processes.

The paper is organized as follows. In Sec. II the observational data and theoretical models of seismic migration and strain waves are presented. Section III contains our model description. In Sec. IV some suitable solutions of the SG equation are extracted. Examples of the application of these solutions to fault dynamics are considered in Sec.V. Section VI contains concluding remarks.

\section{OBSERVATIONAL DATA AND THEORETICAL MODELS}

For an explicit overview of observational data on seismic migration and strain waves, see Refs. [7-13]. Propagation of strain waves is represented quantitatively by the rate of earthquake migration and geophysical responses to active faulting.

Tectonic waves propagating at speeds of $10-100 \mathrm{~km} / \mathrm{yr}$ are detected from migration of large earthquakes [14], temporal variations in seismic wave velocities [11], offsets of water level in wells along faults [15], tilt and ground motions [16], cyclic migration of aseismic gaps in the earth's mantle [12], and oscillatory motion of seismic reflectors $[17,18]$.

Strain waves in faults, with velocities of $1-10 \mathrm{~km} / \mathrm{day}$, are inferred from rapid propagation of remotely triggered seismicity before and after large earthquakes [19], and from radon, electrokinetic, and hydrogeodynamic signals [12]. Geophysical signals have the shape of solitary waves (solitons) and propagate along crustal faults [12].

An important feature of strain waves is the magnitude of the velocity, which is much less (by a factor of $10^{-6}-10^{-7}$ ) than the seismic velocity and much more (by a factor of $10^{6}-10^{7}$ ) than the average velocity of relative motion of tectonic plates. Thus, a quite large collection of observational data provides either explicit or implicit evidence for strain wave propagation in the crust over a range of velocities. A sequence of earthquakes along a fault can be triggered by propagating strain waves generated in the crustal fault during rotation of blocks, along a plate boundary, or at the lithosphere-asthenosphere boundary.

Elsasser [20] proposed a hypothesis of lithospheric stress guides and obtained an equation of local stress transfer in a hard elastic lithosphere superimposed upon a soft viscous asthenosphere. He did not mention waves, and the stress guides are the only indirect indication of the wave mechanism of stress propagation in the "lithosphereasthenosphere" system, with stress diffusing horizontally along the viscous lithosphere-asthenosphere boundary.

Later Bott and Dean [22] applied the ideas of Elsasser [20] to the mechanism of seismicity transfer along plate boundaries. Bott and Dean invoked "stress (strain) waves" propagating along plate edges at speeds of $0.1-100 \mathrm{~km} / \mathrm{yr}$, depending on the wave period and the mechanical properties of lithosphere and asthenosphere, and derived an equation for their velocity.

Lehner et al. [25] further pursued Elsasser's theory of stress diffusion to analyze great earthquake disturbances and to model time-dependent stress alterations at rupturing plate boundaries. The spatial and temporal characteristics of the stress alterations and earthquake patterns they predicted suggest a significant role of viscoelastic lithosphereasthenosphere coupling.

Nikolaevskiy [24] extended Elsasser's model, assuming flexure and compression of a plate rigid over asthenospheric flow, and obtained a rigorous mathematical theory in which tectonic stress propagates as solitary waves, with their energy fed from the stationary asthenospheric flow to compensate for viscous loss. Thus he based the autowave generation mechanism of tectonic waves on the lithosphereasthenosphere system.

Besides the tectonic waves propagating through the lithosphere, strain waves may propagate along crustal and lithospheric faults. The models of Savage [21] and Ida [23] were aimed at explaining seismic migration patterns, including disturbances propagating as stress diffusion at a rate controlled by the response of fault gouge material. Savage [21] described movements of crustal blocks in terms of edge dislocations flowing along a transform fault, in which slip is transferred by creep. He suggested that "creep waves" can be produced by avalanche release of dislocations during an earthquake, which propagate down the transform fault in the direction of dislocation flow. Ida [23] obtained a solution for deformation pulses which move slowly along faults, without change in shape, at a constant velocity. The deformation propagation velocity is controlled mainly by viscosity, thickness, and strength of fault gouge, and ranges from $1-10 \mathrm{~km} /$ day to $10-100 \mathrm{~km} / \mathrm{yr}$.

A closely related topic is the modeling of the temporal and spatial distributions of aftershocks, which is linked to the question of their triggering mechanism. In some cases aftershocks migrate from the main shock with a velocity ranging from $1 \mathrm{~km} / \mathrm{h}$ to $1 \mathrm{~km} / \mathrm{y}$ [26,27]. The temporal distribution of aftershocks obeys a modified Omori law [28], which may be described by a variety of physical mechanisms [29]. The origin of the spatial distribution is more controversial ([30] and references therein). Several researchers have described it as "aftershock diffusion" [27,29,31-34], but there is some doubt that such a mechanism exists [30]. Many models implicitly or explicitly assume that aftershocks are triggered by stress changes although there are alternative approaches [29,35-37]. Recent observations have shown that decay of aftershocks as a function of distance fit an inverse first-power law [38] suggesting that the triggering may be a seismic wave generated by the main shock $[38,39]$.

Recently, the existence of "slow earthquake" has been reported in subduction areas $[40,41]$ as well as at the San Andreas fault [42]. The distinguishing feature of such events is the long duration time (from 2 to 6 orders of magnitude larger than for regular earthquakes). Furthermore, the seismic moment of such events is proportional to the duration of the event, in contrast to regular earthquakes, for which the seismic moment is proportional to the cube of the duration time [43]. The question arises as to the relationship between seismic parameters in such events [44]. Can they be described by the same model as regular earthquakes?

In the last decade the SG equation has been intensively applied to describe phenomena such as the rotation and slid- 
ing of crustal blocks [24,45-49]. The discovery of solitary strain waves in geophysical fields motivated the modeling of rotational oscillations of crustal blocks in faults [24]. Investigation of these waves provides clues to the migration mechanisms of earthquake precursors $[12,24]$ and of tectonic solitary waves [46]. A model of strain waves propagating along a chain of blocks in the earth's crust was proposed by Vikulin [47]. It is assumed that the generation of strain wave has a planetary origin and is connected with the earth's rotation about its axis. A phenomenological model by Bykov $[48,49]$ contains the major factors responsible for the process of unstable sliding (asperities, roughness of contact surfaces) and shows the possibility of the existence of solitary waves of activation of crustal faults, viz. deformation localized at the mesoscopic level and propagating along the fault in the shape of a solitary wave with a velocity which determines a sliding regime along the fault.

In our paper a solution of the SG equation in the form of slow cnoidal waves, i.e., a periodic succession of pulses with a spatial period, has been applied to describe fault dynamics.

\section{MODEL DESCRIPTION}

Macroscopically the friction between plate surfaces governs the local dynamical behavior of the fault. These surfaces always contain roughness (asperities). During plate motion the asperities are deformed. The relative shift of asperities leads to emergence of a restoring force [50]. Thus, the frictional force depends on the relative position of the asperities on the opposing surfaces and changes quasiperiodically. On the other hand, a deformed asperity affects the adjacent asperities on the same plate. So asperities on the same plate interact with each other and with the asperities on the opposite plate. The simplest model of this process is shown in Fig. 1. The masses $M$ represent asperities on the top friction surface. They are constrained to move along an uneven surface. The horizontal and vertical harmonic springs simulate the interaction between asperities on the same and opposite plates, respectively. The upper ends of the vertical springs are fixed in the vertical direction and can move freely in the horizontal direction. The lower surface is considered rigid. This model coincides with the well-known FK phenomenological model describing edge dislocations in crystals [3]. In the FK model the harmonic forces arise due to motion of the ball along the uneven surface in a gravitational field, whereas in our model the harmonic forces arise due to the vertical springs. Since the mathematical descriptions of these two models are identical we will refer to both models as the FK model.

This model has some common features with the BurridgeKnopoff (BK) model [51], which is widely used to simulate spatial and temporal patterns of seismicity and its statistical features, such as large earthquake recurrence, the GutenbergRichter law, foreshock and aftershock activities, and preseismic quiescence [52-58]. The BK model is a chain of blocks coupled to each other by harmonic springs and attached to a fixed surface by flat springs. These blocks are interacting with another surface, moving relative to the fixed one with a given velocity through frictional forces. The dynamic fea-

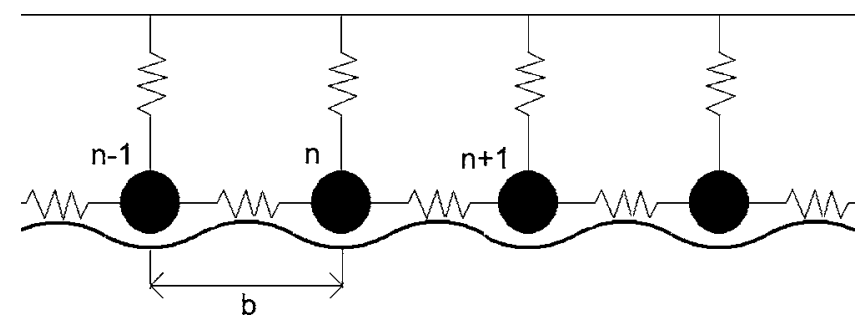

FIG. 1. The model. The balls represent asperities. The sineshaped surface is the opposite plate. The horizontal and vertical harmonic spring model interaction between asperities in the same and opposite plates, respectively. $b$ is the typical distance between neighboring asperities.

tures of the BK chain are defined by the explicit dependence of the frictional force on the velocity of relative plate movement. Complex spasmodic movement of the blocks is obtained by selecting a specific nonlinear relationship between the frictional force and the velocity. In the FK model the nonlinear behavior of the chain is implicit and does not require an explicit frictional force.

Based on this simplified description and the analogy between plasticity in crystal materials and plate movement along a fault we postulate that the latter may be described by the FK model and so by the dimensionless one-dimensional (1D) SG equation,

$$
\frac{\partial^{2} \varphi}{\partial \eta^{2}}-\frac{\partial^{2} \varphi}{\partial \tau^{2}}=\sin \varphi
$$

where $\varphi=2 \pi u / b, \tau=t c A / b$, and $\eta=x A / b$. Here $u$ is the displacement of the plate surface in the $x$ direction (along the fault) relative to the adjusted plate, $b$ is the typical size of asperities in the $x$ direction, $c$ is the velocity of an elastic compressional wave in the earth's crust of density $\rho, t$ is the time, and $A$ is a dimensionless empirical scaling factor which incorporates the roughness between adjusted plates. The derivatives $\varepsilon=\partial \varphi / \partial \eta$ and $w=\partial \varphi / \partial \tau$ are the dimensionless strain ( $x x$ component of strain tensor) and the dimensionless velocity of the asperities in the fault area; $\varepsilon$ and $w$ are in units of $A / 2 \pi$ and $c A / 2 \pi$, respectively. It is also useful to introduce the $x x$ component of the stress tensor $\sigma_{x x} \equiv \sigma$. In the absence of other components of the stress tensor, $\sigma$ can be expressed through $u$ by the formula $\sigma=\rho c^{2} \partial u / \partial x$ [59]. Thus, the dimensionless stress $\sigma=\varepsilon$ is measured in units $\rho c^{2} A / 2 \pi$.

\section{SOLUTIONS OF THE SINE-GORDON EQUATION AND THEIR INTERPRETATION}

The SG Eq. (1) has been actively investigated with regard to a wide spectrum of applications. Here we will extract some solutions, previously obtained elsewhere (see below), which can be used for describing some of the processes of plate movement along a fault.

We will consider first a periodic (traveling wave) solution, which is convenient for determining the relationships between parameters of the model and the parameters of plate movement, and then a nonstationary solution, describing the 
dynamics of a wave train with a large number of oscillations (dislocations).

\section{A. Periodic solution}

Let us consider solutions of the one-dimensional SG equation of the form $\varphi=\varphi(\theta)$, where $\theta=k \xi, \xi=\eta-U \tau, k$ is the wave number measured in units of $A / b$, and $U$ is the wave velocity in units of $c$. Integrating Eq. (1) and restricting ourselves to the periodic solutions with $|U|<1$ we obtain

$$
\begin{gathered}
\varphi=\arcsin [ \pm \operatorname{cn}(-\beta \xi)], \\
\varepsilon \equiv \sigma=2 \beta \operatorname{dn}(\beta \xi), \\
w=U \sigma, \\
\beta=\left[m\left(1-U^{2}\right)\right]^{-1 / 2},
\end{gathered}
$$

where $\operatorname{dn}(\xi, m)$ and $\operatorname{cn}(\xi, m)$ are the elliptic Jacobi functions, $m$ is the modulus of the elliptic function, and $U$ and $m$ are constants $(|U|<1,0 \leq m \leq 1)$. We normalize in such a way that the period of $\theta$ is $2 \pi$ and find that

$$
k=\frac{\pi \beta}{2 K},
$$

where $K(m)$ is the complete elliptic integral of the first kind.

If $m=1$ formula (2) describes a soliton (or kink) solution $[5,6]$ moving along the $x$ axis with speed $U$. In terms of the FK model, a kink is a configuration of $n$ masses (asperities) placed in $n+1$ (or $n-1)$ periods of substrate. This configuration will be referred to as a positive (or negative) dislocation. Let us review some of the features of kinks. Kinks (dislocations) are stable spatially localized formations, which can freely move in either the positive or negative directions with speeds varying from zero to the speed of elastic compressional waves $(|U|=1)$. If a kink passes by some fixed point, the magnitude of $\varphi$ changes by $2 \pi$. In terms of plate movement it means that the passing of one dislocation is equivalent to relative displacement of the plates by one substrate length $b$; positive and negative dislocations correspond to motion of the plates in opposite directions. Kinks of the same sign interact like elastic balls. Kinks with opposite sign attract each other and can pass through each other without change. Under some conditions, kinks of opposite sign can form certain stable and localized configurations known as breathers. The breather can move like a dislocation; however, in contrast to the dislocation, it does not displace the plate after passing. The energy of a breather lies between zero and the energy of two isolated dislocations. The breather has a remarkable property: inside the breather, the total energy is constant, kinetic energy being transformed into potential energy and vice versa (similar to a standing wave on a spring, hence the name "breather" [5]). In the framework of our model it means that relative oscillatory small-scale motion of plates is possible locally.

Solution (2) with $m<1$ is interpreted as an infinite succession of interacting dislocations. In terms of dislocations, the variables $N=k /(2 \pi)$ and $U$ are, respectively, the dimen- sionless density (in units of $A / b$ ) and velocity of dislocations. Let us average, over one oscillation period, the magnitudes of $\sigma, \varepsilon$, and $w$ :

$$
\begin{gathered}
\Sigma \equiv\langle\sigma\rangle=\oint \frac{\sigma d \theta}{2 \pi}=\frac{\pi \beta}{2 K} \equiv k, \quad E \equiv\langle\varepsilon\rangle=\oint \frac{\varepsilon d \theta}{2 \pi}, \\
W \equiv\langle w\rangle=\oint \frac{w d \theta}{2 \pi}=U k .
\end{gathered}
$$

$\Sigma$ (in units of $\rho c^{2} A / 2 \pi$ ), $E$ [scaled by $A /(2 \pi)$ ], and $W$ (in units of $c A / 2 \pi)$ can be interpreted as the average stress, strain, and velocity of relative plate movement, respectively. Note that $N$ can be expressed through the strain by $N$ $=E /(2 \pi)$. Relations (2)-(4) connect important parameters: the wave velocity $U$ and density $N$ of dislocations, the average velocity of plate movement (or particle velocity) $W$, and the average stress $\Sigma$ (or average strain $E$ ).

Since the processes of plate movement are essentially nonstationary, let us consider certain nonstationary solutions of the SG equation, suitable for the investigation of the dynamics of a group of dislocations.

\section{B. Nonstationary solution}

Whitham [4] developed a variational method for the solution of nonlinear dispersive partial differential equations. This method is based on the definition of a system of modulation equations describing slow variations in the parameters of a wave train. This is convenient for the investigation of the dynamics of a wave train with a large number of oscillations (dislocations). For the SG equation, Whitham's method was developed and applied by Forest and McLaughlin [60], Gurevich et al. [61], and Gershenzon [62]. Here we will use the results described by Gershenzon [62]. Whitham's equations, based on the SG equation, are the following $[61,62]$ :

$$
\begin{gathered}
U_{\tau} \frac{\mu}{U^{2}-1}+m_{\tau} \frac{U}{2 m}+U_{\eta} \frac{U \mu}{U^{2}-1}+m_{\eta} \frac{1}{2 m}=0, \\
U_{\tau} \frac{U}{U^{2}-1}+m_{\tau} \frac{\mu}{2 m m_{1}}+U_{\eta} \frac{1}{U^{2}-1}+m_{\eta} \frac{U \mu}{2 m}=0,
\end{gathered}
$$

where $\mu=E / K, m_{1}=1-m$, and $E(m)$ is the complete elliptic integral of the second kind. These equations are valid for $\tau$ $\gg 1$ and $\eta \gg 1$.

We limit our consideration to the "self-similar simple wave" solution. In this case all variables appearing in the solution can be expressed in terms of the modular variable $m$ :

$$
\begin{gathered}
\frac{\eta}{\tau}=V(m), \\
V=\frac{G-\alpha}{G+\alpha}, \quad U=\frac{\delta-\alpha}{\delta+\alpha},
\end{gathered}
$$




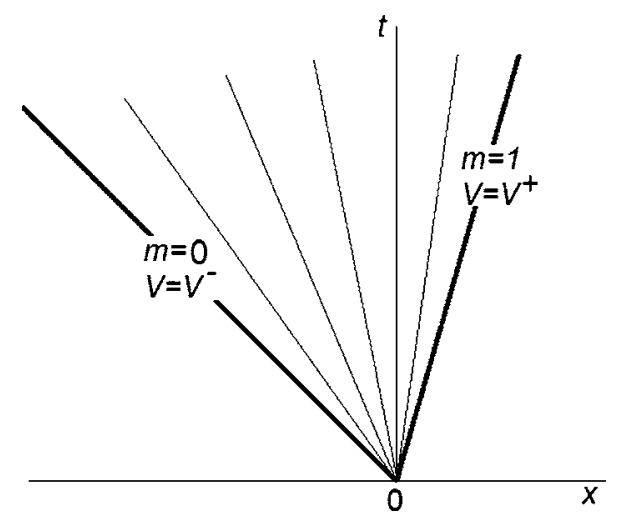

FIG. 2. Isolines of the function $m(\eta, \tau)$. All process parameters (average stress and strain, density of dislocations, and all velocities) are constant along the lines (characteristics) $\eta / \tau=V(m=$ const $)$.

$$
k=E=2 \pi N=\Sigma=\frac{\pi(\delta+\alpha)}{2 K \sqrt{m \delta \alpha}}, \quad W=U k,
$$

where $G=\delta\left(\mu-\sqrt{m_{1}}\right) /\left(\mu+\sqrt{m_{1}}\right), \delta=\left(1-\sqrt{m_{1}}\right)^{2} / m$, and $\alpha$ is a constant $(0<\alpha \leq 1)$ determined by the conditions of the problem (see Sec. V). The variable $V$ is the nonlinear group velocity in units of $c$, i.e., the velocity of an object having a constant value of $m$. For the interpretation of this solution, the $(\eta, \tau)$ plane will be utilized (Fig. 2). Along the lines $\eta / \tau=V=$ constant all variables, including $m$, are constant. The solution is represented by the region expanding in time and limited by the lines $\eta / \tau=V(0)=V^{-}=-1$ and $\eta / \tau=V(1)$ $=V^{+}=(1-\alpha) /(1+\alpha)$. Here and below the indices + and designate the leading and rear edges, respectively. The quantity $V^{+}$is the velocity of the leading edge or the velocity of the first dislocation. To the right side of the line $\eta / \tau=V^{+}$, disturbances are absent $(N=0, W=0$, and $\Sigma=0)$ and waves move into the quiescent region with velocity $V^{+}$.

Another case of interest occurs when $m$ is restricted to values very close to 1 (see Sec. V). In this case, $m_{1} \ll 1$ and formula (6) can be simplified:

$$
\begin{gathered}
U=\nu-m_{1}^{1 / 2}, \\
V=\nu-m_{1}^{1 / 2} \ln \left(m_{1}^{-1 / 2}\right), \\
k=2 \pi N=E=\Sigma=\pi / \ln \left(m_{1}^{-1 / 2}\right), \\
W=\pi\left(\nu-m_{1}^{1 / 2}\right) / \ln \left(m_{1}^{-1 / 2}\right),
\end{gathered}
$$

where $\nu=(1-\alpha) / 2$. We have used the approximation $\ln \left(m_{1}^{-1 / 2}\right) \gg 1$, which is equivalent to the condition $2 N \ll 1$. This means that the distance between dislocations is much larger than the dislocation width.

\section{MODELING OF PLATE MOVEMENT AND ASSOCIATED PHENOMENA: COMPARISON OF ESTIMATES WITH FIELD DATA}

Let us consider the following scenario. We propose that relative plate movement along a fault occurs due to motion of dislocations along the boundary of the plate (here and below the term "dislocation" is used in the sense of the FK model). As a dislocation passes along the length of the plate, it shifts the plate by the length of one substrate period $b$. A dislocation also can relax stress and strain. If the substrate has only small irregularities, dislocations can move steadily under the action of a relatively low external stress. In this case, the plate moves quasicontinuously. This is the creep state. So as a first approximation plate movement can be modeled by the motion of a group of dislocations [63]. The average density of dislocations is proportional to the average strain at the plate boundary. The average velocity of dislocations is the velocity of the corresponding strain wave.

In nature, plate surfaces are usually not uniform and contain irregularities on all scales [64]. Any substrate inhomogeneity tends to impede dislocation motion ("pins" or "traps" them). At a strong trap the dislocations will pile up. This local increase in dislocation density leads to increasing strain energy of their interaction. If some threshold strain value is exceeded, this local quasiequilibrium state becomes unstable and the energy of dislocation interaction transforms into energy of plate movement along the fault. This is the earthquake state.

During an earthquake the dislocation density quickly decreases (process of stress drop); nevertheless it still remains higher than the density outside the focal zone. The consequent diffusion of dislocations (afterslip) triggers aftershocks. We shall assume in this paper that an aftershock is triggered by the stress changes accompanying the propagation of a strain wave. Later in this section we will show that the velocity of diffusion is an almost exponential function of dislocation density (or strain). Thus the decrease in strain with time is reflected in a decrease in the number of aftershocks.

Dislocations of a given sign cannot disappear. After an earthquake they continue to move along the fault, encountering other traps with other thresholds [1], accumulating, and triggering new earthquakes. In the remainder of this section we will show how some quantitative parameters related to these processes can be evaluated by the solutions that we have displayed.

\section{A. Propagation of strain waves}

In the framework of our model, the velocity of a strain wave and the velocity of plate movement are naturally connected. Using the periodic solution [Eqs. (2)-(4)] and returning to dimensional variables we obtain

$$
\begin{gathered}
W=U N b=U E, \\
\Sigma=\frac{W \rho c^{2}}{U} .
\end{gathered}
$$

Note that, in spite of the fact that the relations among $W, U$, and $E$ (or $\Sigma$ ) were obtained from the FK model, they do not contain any "microparameters" of this model since they are parameters averaged over the period of an elliptic function. Nevertheless the stress amplitude $\sigma_{0}$ and strain amplitude $\varepsilon_{0}$ depend on one microparameter, $A$ : 


$$
\sigma_{0}=\rho c^{2} \varepsilon_{0}=\frac{\beta c^{2} A \rho}{\pi} \approx \frac{c^{2} \rho A}{\pi} .
$$

The last approximation is valid if $U \ll c$ and $m_{1} \ll 1$, which almost always is the case. In this situation, the values of $\sigma_{0}$ and $\varepsilon_{0}$ do not depend on any process parameters such as the velocity of a process and the dislocation density. They depend on the model parameters only, such as $c, \rho$, and $A$. In particular, it means that there are "maximal" values of stress and strain which the crustal material is able to handle without destruction. Indeed, from the point of view of the FK model the stress $\sigma_{0}$ is exactly the stress needed to move uniformly all the balls across of the sine-shaped surface (see Fig. 1), which means that the balls (or upper surface) could move freely relative to the bottom surface (crustal failure). Thus, the quantities $\varepsilon_{0}$ and $\sigma_{0}$ could be interpreted as the maximal values for a fault with the above material parameters.

Let us estimate the typical values of strain and stress. Using a plate movement velocity $W=3 \mathrm{~cm} /$ year, strain wave velocity $U=(10-100) \mathrm{km} /$ year, crustal density $\rho=3$ $\times 10^{3} \mathrm{~kg} / \mathrm{m}^{3}$, elastic compressional wave velocity $c=6$ $\times 10^{3} \mathrm{~m} / \mathrm{s}$, and "microfriction" parameter $A=10^{-3}$ (this value will be estimated below), we obtain from formulas (8) and (9): average strain $E=3 \times\left(10^{-6}-10^{-7}\right)$, average stress $\Sigma=0.3-3$ bar, maximal strain $\varepsilon_{0}=2 \times 10^{-4}$, and maximal stress $\sigma_{0}=200$ bar. We see that for stationary plate movement, relatively small values of average stress and strain are needed. Local stress and strain values (at the maximum of the elliptic function) are much higher but still reasonable. This is consistent with the fact that during frictional processes the main stress occurs at only a few spots.

\section{B. Earthquakes}

The appearance and propagation of rupture is an important part of fault dynamics. A rupture arises due to stress accumulation during relative plate movement. On the other hand, a rupture itself causes an acceleration of inelastic stress and strain propagations along the fault. In the framework of our model, the accumulation and release of stress are equivalent to the accumulation and take-off run of dislocations. In what follows, the self-similar simple wave solution obtained earlier will be used for modeling the propagation of rupture.

As initial conditions $(\tau=0)$, we consider that $\eta=0$ divides two areas of different dislocation density and velocity:

$$
\begin{gathered}
N(\eta<0, \tau=0)=N^{-}, \quad U(\eta<0, \tau=0)=U^{-}, \\
N(\eta>0, \tau=0)=N^{+}<N^{-}, \quad U(\eta>0, \tau=0) U^{+} .
\end{gathered}
$$

What is time evolution of this system? It is described by dimensionless formulas (5) and (6)

$$
\alpha=\delta^{-} \frac{1-U^{-}}{1+U^{-}}, \quad \delta^{-} \equiv \delta\left(m^{-}\right),
$$

where $m^{-}$is defined from the relation

$$
N^{-}=\left\{4 K^{-}\left\{m^{-}\left[1-\left(U^{-}\right)^{2}\right\}^{1 / 2}\right\}^{-1}, \quad K^{-} \equiv K\left(m^{-}\right) .\right.
$$

Formulas for the velocity of the leading and rear edges are

$$
\begin{array}{ll}
V^{-}=\left(G^{-}-\alpha\right) /\left(G^{-}+\alpha\right), & G^{-} \equiv G\left(m^{-}\right), \\
V^{+}=\left(G^{+}-\alpha\right) /\left(G^{+}+\alpha\right), & G^{+} \equiv G\left(m^{+}\right),
\end{array}
$$

where $m^{+}$is defined from the expression

$$
N^{+}=\frac{\delta^{+}+\alpha}{4 K^{+}\left(m^{+} \delta^{+} \alpha\right)^{1 / 2}}, \quad K^{+} \equiv K\left(m^{+}\right), \quad \delta^{+} \equiv \delta\left(m^{+}\right),
$$

with $\alpha$ from Eq. (10).

If $N^{+} \ll 1$ and $N^{-} \ll 1$ formulas (10)-(13) are simplified, and we find

$$
\begin{gathered}
\frac{1-\alpha}{2}=U^{-}+e^{-1 /\left(2 N^{-}\right)}, \quad m_{1}^{-}=e^{-1 / N^{-}}, \\
V^{-}=U^{-}-\left(2 N^{-}\right)^{-1} e^{-1 /\left(2 N^{-}\right)}, \\
V^{+}=U^{-}+e^{-1 /\left(2 N^{-}\right)}-\left(2 N^{+}\right)^{-1} e^{-1 /\left(2 N^{+}\right)} .
\end{gathered}
$$

So relations (5), (6), and (10)-(13) in the general case and Eqs. (5), (7), (14), and (15) in the case $N \ll 1$ provide the solution to this problem. These solutions are suitable for modeling earthquake dynamics. We need to find a minimal set of macroparameters whose values can be obtained from experiment, in term of which we can determine all other parameters and describe the dynamics of the process. It is natural to assume that $U^{-}=0$ and $N^{+}=0$ since before the earthquake the dislocation velocity inside the focal zone $\left(U^{-}\right)$ and the dislocation density outside the focal zone $\left(N^{+}\right)$are presumed small. If we choose only one specified macroparameter, for example, the speed of rupture propagation $\left(V^{-}\right.$in our model), then the remaining parameters may be obtained (in dimensionless form) using the various formulas. Let us illustrate how this works. First we express $V^{-}$in dimensionless units using the given model parameter $c$. With the condition $U^{-}=0$ and $N^{+}=0$, formulas (10) and (12) reduce to

$$
\begin{gathered}
\alpha=\delta^{-}, \quad \delta^{-} \equiv \delta\left(m^{-}\right), \quad \text { and } \\
V^{-}=\left(G^{-}-\delta^{-}\right) /\left(G^{-}+\delta^{-}\right), \quad G^{-} \equiv G\left(m^{-}\right) .
\end{gathered}
$$

These allow us to find $m^{-}$from $V^{-}$. Knowing $m^{-}$then allows us to obtain the density of dislocations in the pileup $\left(N^{-}\right)$, the initial stress $\left(\Sigma^{-}\right)$, and/or the strain $\left(E^{-}\right)$in the focal area immediately before the earthquake, as well as the velocity of propagation of the front edge $\left(V^{+}\right)$, from

$$
\begin{gathered}
N^{-}=\Sigma^{-} /(2 \pi)=E^{-} /(2 \pi)=\left\{4 K^{-}\left(m^{-}\right)^{1 / 2}\right\}^{-1}, \quad K^{-} \equiv K\left(m^{-}\right), \\
V^{+}=(1-\alpha) /(1+\alpha), \quad \alpha \equiv \delta\left(m^{-}\right),
\end{gathered}
$$

derived from formulas (6), (11), and (12). Figure 3 depicts the dependence of $V^{-}, \Sigma^{-}, E^{-}, \alpha$, and $V^{+}$on $m^{-}$. From this figure one can find all these parameters from the given value of $V^{-}$and then can describe quantitatively the dynamic behavior of the rupture process using formula (6). Figure 4 depicts the spatial distribution of the variables, $V(x), U(x)$, $W(x), \Sigma(x)$, and $E(x)$ (in dimensionless units), in the case $V^{-}=0.5 c$ for $x$ in units of $0.5 c t$ at any time in the interval $0<t<T$, where $T$ is the rise time. 

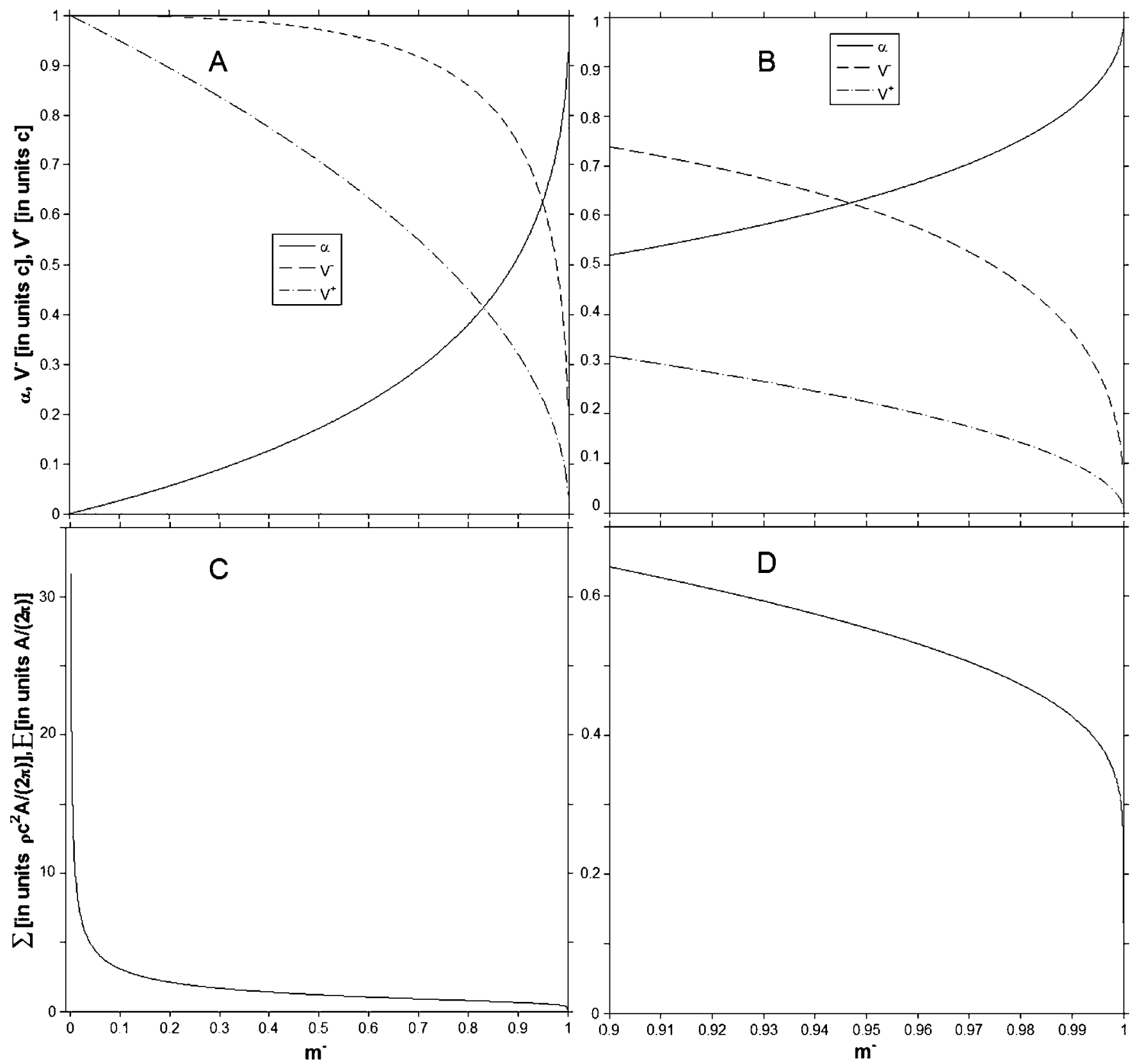

FIG. 3. The dependence of model variables $\left(\alpha, V^{-}, V^{+}, \Sigma / E\right)$ on $m^{-}$, the elliptic function modulus for the rear edge of a disturbance. Graphs $(\mathbf{B})$ and $(\mathbf{D})$ show the variables on an expanded scale near $m^{-}=1$.

We see that a disturbance (rupture) propagates in both directions away from the epicenter; however it runs much faster in the direction of the largest dislocation density, indeed $\left|V^{-}\right| \gg\left|V^{+}\right|$and $\left|x^{-}\right| \gg\left|x^{+}\right|$if $N^{-} \gg N^{+}$and $U^{-}=0$ [see Fig. 4(a) and formula (15)]. The rupture continues to propagate until the rear edge reaches the end of the pileup. The extent of the pileup is the focal zone. The time of passing through the focus is the "rise time" $T=l^{-} / V^{-}$, where $l^{-}$is the focal size. The size of the rupture zone in opposite direction is

$$
l^{+}=V^{+} T=V^{+} l^{-} / V^{-} .
$$

All dislocations in the disturbed area move in one direction (from the left to the right in Fig. 4) and $U(x)$ is positive for all $x$ [Fig. 4(a)]. The velocity of dislocations is a monotonic function of $x$ and changes from $U^{-}=0$ to $U^{+}=V^{+}$. The strike slip velocity (particle velocity) $W$ also has constant sign, however, in contrast to $U$, the function $W(x)$ has a maximum at the epicenter and vanishes at the boundaries of the disturbed area [Fig. 4(b)]. After the rupture the internal stress and dislocation density inside the focus are lower than their initial values but still higher than outside the focal zone. It means that only part of the initial stress drops as a result of the earthquake. Taking into account that the total number of dislocations is conserved and using relations (6) and (10), we find the magnitudes of the relative stress drop $\Delta$, 


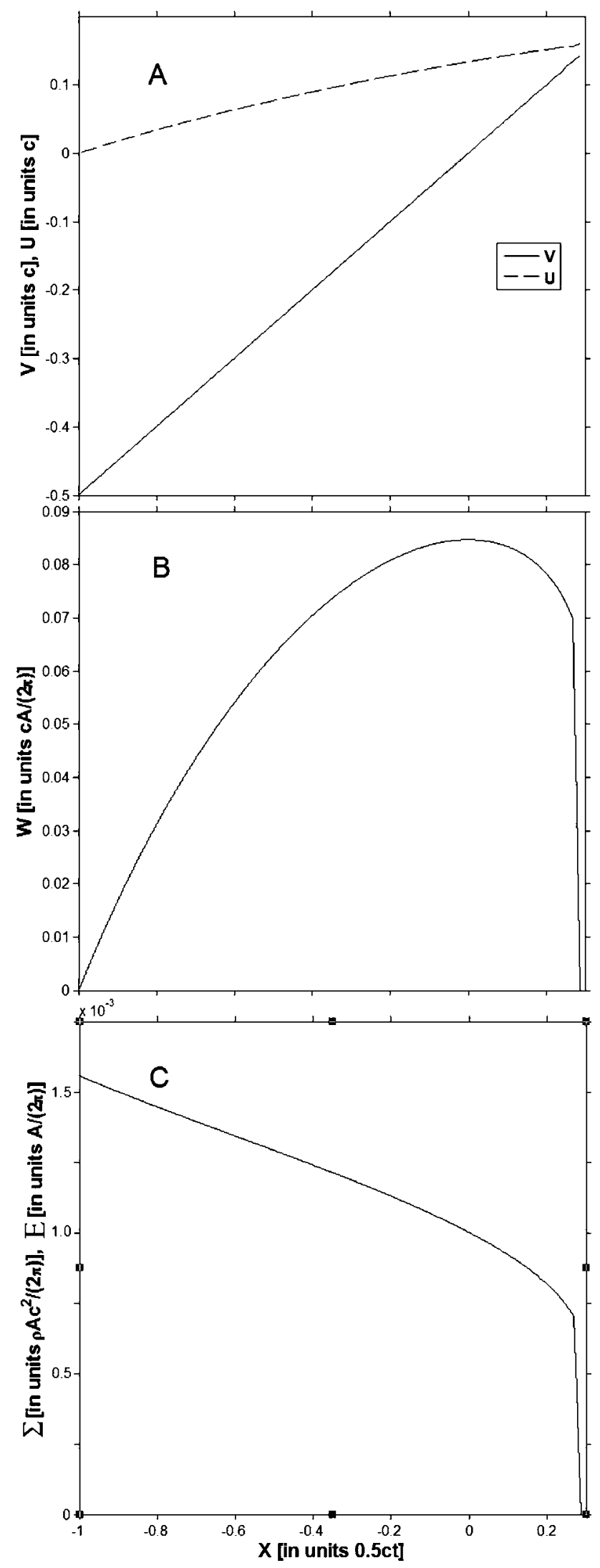

FIG. 4. Spatial distribution $x$ of (A) the group velocity $V(x)$ and dislocation velocity $U(x),(\mathbf{B})$ particle (or plate) velocity $W(x),(\mathbf{C})$ stress $\Sigma(x)$, and strain $E(x)$, at a given time $t(0<t<T)$ during an earthquake. All variables are in dimensionless units.
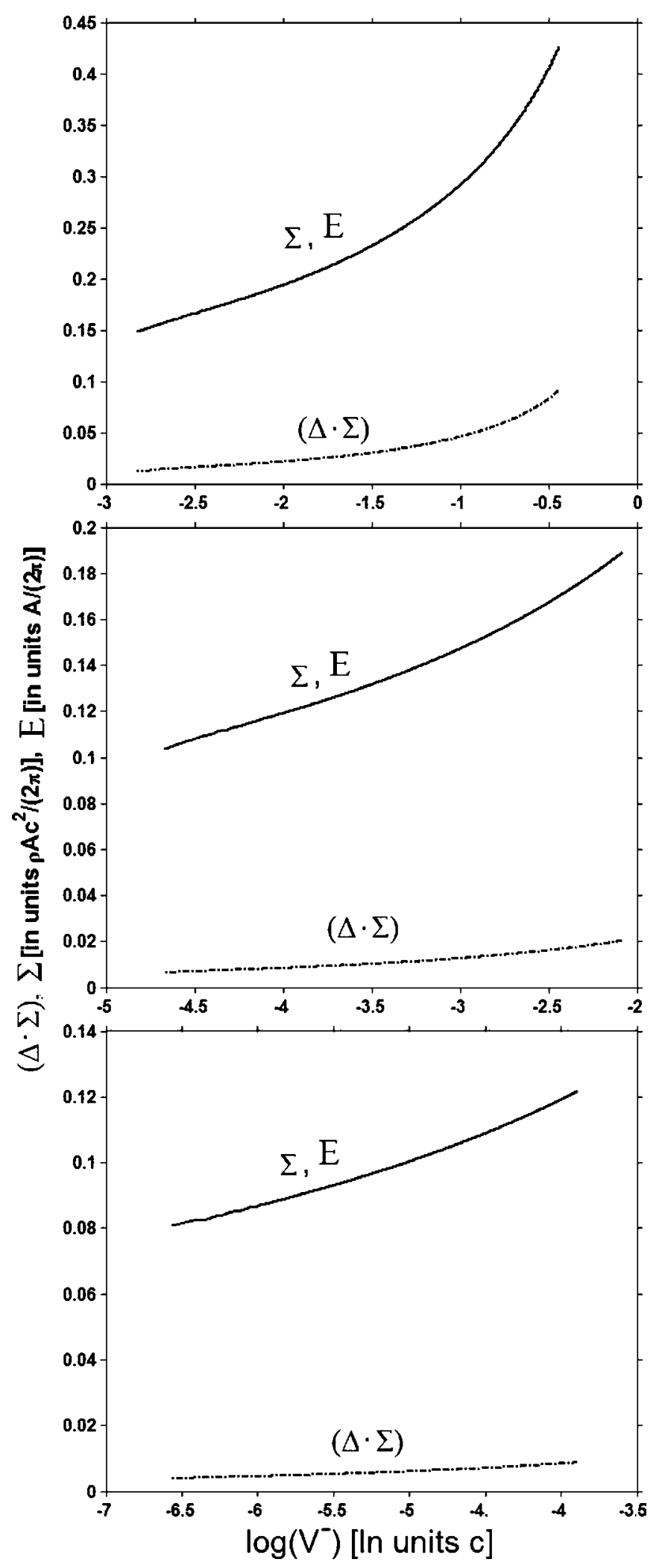

FIG. 5. The preseismic stress $\Sigma$, strain $E$, and postseismic stress drop $\Delta \Sigma$ as functions of rapture velocity. All variables are in dimensionless units. The stress, strain, and stress drop vary insignificantly for a wide range of seismic events, from very slow slips to regular earthquakes. 
TABLE I. The calculated values of earthquake and afterslip parameters for the Imperial Valley earthquake of 1979 .

\begin{tabular}{ccccccc}
\hline \hline Time $(\mathrm{t})$ & $V^{+}$ & $10^{-5} E$ & $\begin{array}{c}\sum \\
(\mathrm{bar})\end{array}$ & $\begin{array}{c}\delta \Sigma \\
(\mathrm{bar})\end{array}$ & $\begin{array}{c}x^{+} \\
(\mathrm{km})\end{array}$ & $\begin{array}{c}S(x=0) \\
(\mathrm{m})\end{array}$ \\
\hline 0 & $1 \mathrm{~km} / \mathrm{s}$ & 8 & 88.5 & 0 & 0 & 0 \\
$12 \mathrm{~s}$ & $1 \mathrm{~km} / \mathrm{s}$ & 6 & 67.5 & 21 & 11 & 1 \\
1 day & $11 \mathrm{~km} /$ day & 2.3 & 25 & 63.5 & 97 & 1.9 \\
1 month & $11 \mathrm{~km} /$ month & 1.6 & 18 & 70.5 & 134 & 2.2 \\
1 year & $11 \mathrm{~km} /$ year & 1.2 & 13.5 & 75 & 162 & 2.4 \\
\hline \hline
\end{tabular}

$$
\Delta=\frac{\mu^{-}}{1+\mu^{-}}, \quad \mu^{-} \equiv \mu\left(m^{-}\right) .
$$

So far all our calculated parameters are in dimensionless form. Some can be converted to dimensioned forming using our model parameters $c$ and $\rho$. But others, such as the strain $\varepsilon$, stress $\Sigma$ and absolute value of stress drop $\delta \Sigma$, require a knowledge of the microfriction parameter $A$. Another such quantity is $S(x, t)$, the relative displacement of the plate boundaries. It is given by

$$
\begin{aligned}
S(t, x) & =\int_{0}^{t} W\left[m\left(t^{\prime}, x\right)\right] d t^{\prime} \\
& =\int_{0}^{t} \pi c A\left(\delta-\delta^{-}\right)(4 K)^{-1}\left(m \delta \delta^{-}\right)^{-1 / 2} d t^{\prime} .
\end{aligned}
$$

Here $m(t, x)$ is expressed through $x$ and $t$ by using relation (5). At the epicenter $x=0$, the particle velocity is constant in time since $m(x=0)=$ const and $V(x=0)=0$. In this case the expression for the displacement is simplified to $S(0, t)$ $=W(x=0) t$.

In order to find the value of $A$ we need to know the value of one more macroparameter (besides $V^{-}$) such as the velocity of plate movement during the earthquake $[W(x=0)]$ or the strain in the focal zone immediately before the earthquake $[E(t=0, x=0)]$ :

$$
\begin{gathered}
A=2 \pi E(t=0, x=0) / E(\tau=0, \eta=0), \\
A=2 \pi W(x=0) /[c W(\eta=0)],
\end{gathered}
$$

where $E(t=0, x=0)$ is unscaled and $W(x=0)$ is dimensioned, and $E(\tau=0, \eta=0)$ is scaled and $W(\eta=0)$ is dimensionless and expressed thorough $V^{-}$by formulas (6), (10), and (12).

Let us check our model using data from the Imperial Valley earthquake (1979) (Table I), which has been investigated rather thoroughly. As initial data we will take $V^{-}=0.5 c$ as the averaged rupture velocity and the size of the rupture zone $l^{-}=35 \mathrm{~km}$ or, more precisely, the extent of the rupture from the epicenter to the north end of the rupture [65]. The parameters $m_{1}^{-}=0.02568, \quad N^{-}=0.156, \quad T=12 \mathrm{~s}, \quad V^{+}=0.16 c$ $=960 \mathrm{~m} / \mathrm{s}, l^{+}=11 \mathrm{~km}$, and $\Delta=0.24$ can be found from formulas (6), (10)-(12), (16), and (17). We can now obtain the parameter $A$. Assuming $S(x=0)=1 \mathrm{~m}$ (see [65]) we calculate $W(x=0)=S(x=0) / T=0.086 \mathrm{~m} / \mathrm{s}$ and $A=10^{-3}$ using the second formula of Eq. (19) and the value $W(\eta=0)=0.085$ ob- tained from Fig. 4(b). Now we can find the initial values of strain and stress, $E=0.8 \times 10^{-4}$ and $\Sigma=88.5$ bar, and the stress drop $\delta \Sigma \equiv E \Delta=21$ bar (see Table I). The magnitudes of the calculated earthquake parameters are in reasonable agreement with values obtained from the faulting model based on the near-source strong motion data $[64,65]$.

\section{Afterslip}

After the earthquake, dislocations continue to move from the focal area and stress continues to relax. Aftershock and afterslip activities reflect these processes. The quantity $V^{+}$ defines the velocity of inelastic stress relaxation or the velocity of propagation of the disturbance. The value of $V^{+}$ could be much smaller than the velocity of elastic compressional waves $c$. Note that $\left|V^{-}\right| \gg\left|V^{+}\right|$if $N^{+} \ll N^{-} \ll 1$ [see Eq. (15)]. In particular, it means that the disturbance propagates faster along the more highly stressed portion of the fault. It means also that, as a result of dislocation diffusion through the boundaries of the region of high dislocation density, the pileup slowly spreads. During this time the dislocation density (and stress) decreases slowly, but almost uniformly, along the pileup.

Let us find some afterslip parameters. The formulas obtained above exhibit strong spiking and are suited for the description of rupture. However, we can also use them for the approximate description of the postseismic process. Since the number of dislocations is conserved, we can write

$$
N_{p}(t)\left[l_{0}(1+\Delta)+\int_{T}^{t} V^{+}\left(t^{\prime}\right) d t^{\prime}\right]=N^{-} l_{0},
$$

where $N_{p}(t)$ is the density of dislocations averaged along the pileup. The relation $N_{p} \ll 1$ will be satisfied shortly after the earthquake occurs $(t \gg T)$. In this case we can use an approximate expression for $V^{+}: V^{+}=e^{-1 /\left(2 N_{p}\right)}$ [second formula of Eq. (15)]. Then Eq. (20) leads to the following expressions:

$$
N_{p}=(2 \ln \psi)^{-1}, \quad V^{+}=c \psi^{-1}, \quad \text { and } \quad x^{+}=2 N^{-} l_{0} \ln \psi,
$$

where

$$
\psi=e^{(1+\Delta) / 2 N^{-}}+\frac{c(t-T)}{2 N^{-} l_{0}} \quad \text { and } \quad t \gg T .
$$

The expression for the magnitude of $S$ after the earthquake is 


$$
S(x, t)=\int_{t^{*}}^{t} A c N_{p} U d t^{\prime}, \quad \text { where } t^{*}=T e^{x /\left(2 N{ }^{-} l_{0}\right)} .
$$

For the case $N^{+} \ll N_{p} \ll 1, U$ is an almost linear function of $x$ :

$$
U \approx V^{+}\left(x+l^{-}\right) /\left(x^{+}+l^{-}\right) .
$$

Formula (22) with the parameter $U$ given by Eq. (23) and $N_{p}$ and $x^{+}$given by Eq. (21) defines the spatial and temporal distribution of $S$ after the earthquake.

Using the data from the Imperial Valley earthquake [65] and relations (21)-(23) we can estimate the magnitude of the afterslip parameters $V^{+}, E, \Sigma, \delta \Sigma, x^{+}$, and $S(x=0)$ for one day, one month, and one year after the earthquake (see Table I). The first two rows in the table contain the values of the parameters before and immediately after the earthquake.

From expression (21) it follows that the velocity of inelastic stress propagation after an earthquake is proportional to $1 /($ const $+t)$. The number of aftershocks $n(t)$ occurring (per unit time) at time $t$ obey the modified Omori law $n(t)$ $=b /(\text { const }+t)^{q}[28]$, where $a$ and $q$ are some constants and $q$ ranges from 0.5 to 2 . Assuming that the strain wave triggers aftershocks, one may conclude that our finding is consistent with Omori's law.

The velocity of inelastic stress propagation depends on the size of the focal zone as well as the initial stresses [second formula of Eq. (21)] if $t \gg T+\frac{2 N^{-} l_{0}}{c} e^{(1+\Delta) / 2 N^{-}} \approx 10^{2}$ s. So the value of $V^{+}$decreases from $1 \mathrm{~km} / \mathrm{s}$ just after the earthquake to $10 \mathrm{~km} /$ year one year after (see second column in TableI). The stress (dislocation density) relaxes very slowly after the earthquake. After one year the stress and strain have decreased to only about one-seventh of their initial values. The size of the disturbed area also increases very slowly.

It was shown recently [38] that the spatial decay of aftershock density is proportional to an inverse first-power law. Our model is consistent with this finding. Indeed, from the first and the last formulas in Eq. (21) we find that $N_{p}$ $\sim 1 / x^{+}$. Since stress is proportional to dislocation density, and with an assumption that the spatial density of aftershocks is proportional to the stress change, we obtain aforementioned power law.

\section{Slow earthquakes}

The so-called slow earthquakes include tremor, lowfrequency earthquakes, very low-frequency earthquakes, and silent earthquakes [see [43] and references therein]. In addition to features already mentioned in Sec. I, these slow events are accompanied by a lower stress drop (up to 2 orders of magnitude less) with about the same seismic moment as regular earthquakes. Slow events occur only in the subduction zone or in the lower part of the crust, where friction parameters could be different from the more rigid upper crust. Can our model describe dynamics of slow earthquakes?

Supposing as before that $U^{-}=0$ and using the relations $\Sigma^{-}=E^{-}=2 \pi N^{-}$, we can find from formulas (10)-(12) and (17) the relationship between rupture velocity and initial accumulated stress as well as stress drop after the event (see Fig. 5). From this figure one can see that when the rupture velocity is reduced by 6 orders of magnitude (from $0.5 c$ to $5 \times 10^{-7} c$ ) the value of initial stress varies only by a factor of 6 , the relative stress drop by a factor of 5 (from 0.24 to 0.05 ), and so the absolute value of stress drop by a factor of 30 . The about the same level of stress buildup could cause ether a regular earthquake or slow event. However, a slow event still requires a little less energy buildup. In our model, the accumulated stress and stress drop could be estimated by any two known experimental parameters, for example, rupture and particle velocities. Experimental data for slow earthquakes are presented in Ref. [43]. Using these data we can calculate the average rupture and particle velocities and estimate the initial accumulated stress and strain and stress drop using our model. The results are presented at Table II. Column 4 contains the experimental stress drop $(\Delta \Sigma)$ estimated by the relation $\Delta \Sigma \approx \mu_{0} D / L$, where $\mu_{0}=\rho c_{s}^{2}$ and $\rho=3 \times 10^{3} \mathrm{~kg} / \mathrm{m}^{3}$,

TABLE II. The parameters of slow earthquakes $(T, D$, and $L$ are, respectively, the characteristic time of event, the averaged amount of slip, and dimension of the fault plane; SSE: slow slip event, ETS: episodic tremor and slip), (column 1-3) from [43]; experimental stress

\begin{tabular}{|c|c|c|c|c|c|c|c|}
\hline & 1 & 2 & 3 & 4 & 5 & 6 & 7 \\
\hline Type & $\begin{array}{c}T \\
\text { (s) }\end{array}$ & $\begin{array}{c}D \\
(\mathrm{~m})\end{array}$ & $\begin{array}{c}L \\
(\mathrm{~m})\end{array}$ & $\begin{array}{l}\text { Stress } \\
\text { drop } \\
\text { (bar) }\end{array}$ & $\begin{array}{c}\text { Accumulated } \\
\text { stress (bar) } \\
\text { model }\end{array}$ & $\begin{array}{l}\text { Stress drop } \\
\text { (bar) } \\
\text { model }\end{array}$ & $\begin{array}{c}\text { Accumulated } \\
\text { strain } \\
\text { model }\end{array}$ \\
\hline SSE short-term & $3-6 \times 10^{5}$ & $0.008-0.026$ & $3-5 \times 10^{4}$ & $0.17-0.32$ & 6 & 0.37 & $0.32 \times 10^{-5}$ \\
\hline SSE long-term & $10^{7}$ & 0.11 & $5-7 \times 10^{4}$ & 1.2 & 50 & 2.5 & $2.6 \times 10^{-5}$ \\
\hline \multirow[t]{3}{*}{ Silent earthquakes } & $3 \times 10^{7}$ & $<0.18$ & $6 \times 10^{4}$ & 1.9 & 143 & 6.9 & $7.5 \times 10^{-5}$ \\
\hline & $5 \times 10^{7}$ & 0.2 & $10^{5}$ & 1.26 & 95 & 4.6 & $5 \times 10^{-5}$ \\
\hline & $1.7 \times 10^{7}$ & 0.1 & $2-5 \times 10^{5}$ & 0.18 & 6.1 & 0.33 & $0.32 \times 10^{-5}$ \\
\hline ETS & $2-3 \times 10^{6}$ & 0.02 & $1.5 \times 10^{5}$ & 0.084 & 2.5 & 0.15 & $0.13 \times 10^{-5}$ \\
\hline Afterslip & $3 \times 10^{7}$ & $0.7-0.9$ & $10^{5}$ & 5 & 276 & 13.7 & $14.4 \times 10^{-5}$ \\
\hline Slow slip (creep) & $2 \times 10^{5}$ & $0.03-0.1$ & 5,000 & 8.2 & 264 & 14.5 & $13.8 \times 10^{-5}$ \\
\hline Slow slip in volcano & $1.9 \times 10^{5}$ & $>0.015$ & $1.5 \times 10^{4}$ & 0.6 & 17 & 1 & $0.9 \times 10^{-5}$ \\
\hline
\end{tabular}
drop (column 4); modeled accumulated stress before events (column 5); modeled stress drop (column 6); modeled accumulated strain (7). 
$c=8 \times 10^{3} \mathrm{~m} / \mathrm{s}$, and $c / c_{s}=1.75$. Here $c_{s}$ is the shear velocity, $D$ is the average amount of slip, and $L$ is the dimension of the fault plane. Column 6 shows the calculated values of stress drop using our model. Although the modeled values are in reasonable agreement with experimental values, the former is larger by a factor of 1.5-3. For some events (see rows 5 and 7) the estimated initial stress and strain are too high, suggesting that the approximation of constant propagation velocity does not apply for some slow events. In this case more realistic data could be used, e.g., the values of rupture and particle velocities as function of time. The overall we consider that the proposed model could be used for a quantitative description of slow earthquake processes.

\section{CONCLUSIONS}

(1) The 1D Frenkel-Kontorova (FK) model has been used for description of the plate movement along transform faults. It is assumed that the surfaces of the plate boundaries are uniform but contain spatial heterogeneity (asperities). These asperities, under external stress, cause the appearance of dislocations. Relative displacement of the plate boundaries is realized due to the movement of these dislocations. Such motion of rough plate surfaces requires much less external stress than spatially uniform movement.

(2) In the continuum limit the FK model is described by the sine-Gordon equation. Here we have used only the solutions representing kinks (of one sign) to model a group of dislocations. The formulas obtained are suitable for describing strain waves, earthquakes, and afterslip.

(3) In the framework of the continuum FK model, the fundamental velocities, inelastic strain waves $V$, and plate movement $W$ are naturally connected by Eqs. (8), (10), and (12). And the magnitudes of both $W$ and $V$ cannot exceed the third fundamental velocity, $c$, of elastic compressional waves. Note that these relations do not contain any microparameters of the model.

(4) In terms of the model considered here, a wave of inelastic stress disturbance (a hyperbolic wave, in contrast to an Elsasser diffusion wave) could propagate along a fault with any velocity from zero up to the velocity of elastic compressional waves. The magnitude of the velocity is a strong (almost exponential) function of stress or strain and changes from a few $\mathrm{km} / \mathrm{s}$ during earthquakes to a few dozen $\mathrm{km}$ per day, month, or year during afterslip and interearthquake periods.

(5) Formulas (5)-(7), (10)-(18), and (21)-(23) describe the dynamics of earthquake and afterslip, respectively. It is sufficient to specify two macroparameters, for example, the strike-propagation velocity and the particle velocity (which can be found from observations of the size of the rupture zone and plate displacement, respectively, together with the observed rupture time) and the model parameters of crustal density and elastic compressional wave velocity, to calculate such process parameters as the initial stress and strain, the stress drop, and the postseismic temporal and spatial distributions of the stress, strain, particle velocity $W(x, t)$, displacement $S(x, t)$, and velocity of inelastic stress propagation $V(x, t)$. The values of the calculated parameters for the Imperial Valley earthquake of 1979 are in reasonable agreement with values obtained from measurements.

(6) The velocity of inelastic stress propagation after an earthquake is proportional to $1 /($ const $+t)$. The temporal distribution of aftershocks, $n(t)=a /(\text { const }+t)^{q}$ (modified Omori's law [28]), shows the same time dependence (for $p$ $=1$ ) suggesting that aftershocks are triggered by strain waves generated by the earthquake. Our model is also consistent with the spatial decay of aftershock density being proportional to an inverse first power of distance from the main shock [38].

(7) One of the basic solutions of the SG equation is a breather. Thus the model predicts the possibility of the existence of some local formations with internal motion. As a result of this motion, the local relative plate displacement changes sign periodically in time and space. A breather is formed, under certain conditions, by the interaction of two dislocations of opposite sign. Lattice impurities (in our case substrate heterogeneities) interact with breathers, absorbing them [66]. Breathers dissipate with time, losing their strain energy via seismic radiation. The breather entity appears to be the almost the same thing as a quasidislocation of null Burgers vector $[67,68]$.

The presence of breathers in fault dynamics may have been observed after the Loma Prieta earthquake (1989). Indeed, an unusual behavior of the aftershocks was noted. There were large numbers of right-lateral, left-lateral, reverse, and normal faulting aftershocks [69-71]: in many locations, seemingly incompatible types of earthquakes occurred in approximately the same place (citation from [71]). The phenomenon finds a possible explanation via the breather solution.

(8) Our model does not consider energy dissipation which accompanies plate movement. Other solutions of the SG equation are breathers and phonons. Breather energy could be transferred to the phonons and eventually to the heat. Thus there is a possibility to describe transformation of the elastic energy to the heat in the framework of the continuum FK model.

\section{ACKNOWLEDGMENTS}

The authors gratefully acknowledge A. Krylov and B. Birger for fruitful discussions, and J. Savage, J. Weertman, and the anonymous reviewers for useful comments. 
[1] J. C. Savage, J. L. Svarc, and Y. Shui-Beih, J. Geophys. Res. 110, B11402 (2005).

[2] J. P. Hirth and J. Lothe, Theory of Dislocations (McGraw-Hill, New York, 1982).

[3] Ya. I. Frenkel' and T. A. Kontorova, Sov. Phys. JETP 8, 89 (1938).

[4] G. B. Whitham, Linear and Nonlinear Waves (Wiley, New York, 1974).

[5] G. L. Lamb, Elements of Soliton Theory (John Wiley, New York, 1980).

[6] R. K. Dodd, J. C. Eilbeck, J. D. Gibbon, and H. C. Morris, Solitons and Nonlinear Wave Equations (Academic Press, London, 1982).

[7] K. Mogi, Bull. Earthquake Res. Inst., Univ. Tokyo 46, 53 (1968).

[8] K. Kasahara, Tectonophysics 52, 329 (1979).

[9] I. L. Nersesov, A. A. Lukk, V. I. Zhuravlev, and O. N. Galaganov, Izv. Akad. Nauk. SSSR, Fizika Zemli 5, 102 (1990).

[10] V. L. Barabanov, A. O. Grinevskiy, V. M. Belikov, and G. A. Ishankuliev, in Dynamic Processes in the Geophysical Medium, edited by A. V. Nikolaev (Nauka, Moscow, 1994), pp. 149-167.

[11] M. V. Nevskiy, in Dynamic Processes in the Geophysical Medium, edited by A. V. Nikolaev (Nauka, Moscow, 1994), pp. 40-55.

[12] V. N. Nikolaevskiy, in Localization and Bifurcation Theory for Soils and Rocks, edited by T. Adachi, F. Oka, and A. Yashima (A. A. Balkema, Rotterdam, 1998), pp. 137-142.

[13] V. G. Bykov, Russ. Geol. Geophys. 46, 1176 (2005).

[14] R. S. Stein, A. A. Barka, and J. H. Dieterich, Geophys. J. Int. 128, 594 (1997).

[15] V. L. Barabanov, A. O. Grinevsky, I. G. Kissin, and M. R. Mil'kis, Izv. Akad. Nauk. SSSR, Fizika Zemli 5, 21 (1988).

[16] H. Ishii, A. Takagi, and S. Suzuki, Gerlands Beitr. Geophys. 88, 163 (1979).

[17] T. A. Bazavluk and F. N. Yudakhin, Dokl. Akad. Nauk 329, 565 (1993).

[18] V. A. Bormotov and V. G. Bykov, Geol. Pac. Ocean 16, 981 (2001).

[19] D. P. Hill, M. J. S. Johnston, J. O. Langbein, and R. Bilham, J. Geophys. Res. 100, 12985 (1995).

[20] W. M. Elsasser, in The Application of Modern Physics to the Earth and Planetary Interiors, edited by S. K. Runcorn (Wiley-Interscience, New York, 1969), pp. 223-246.

[21] J. C. Savage, J. Geophys. Res. 76, 1954 (1971).

[22] M. H. P. Bott and D. S. Dean, Nature (London) 243, 339 (1973).

[23] Y. Ida, Phys. Earth Planet. Inter. 9, 328 (1974).

[24] V. N. Nikolaevskiy, Geomechanics and Fluidodynamics (Kluwer, Dordrecht, 1996).

[25] F. K. Lehner, V. C. Li, and J. R. Rice, J. Geophys. Res. 86, 6155 (1981).

[26] E. Jacques, J. C. Ruegg, J. C. Lepine, P. Tapponnier, G. C. P. King, and A. Omar, Geophys. J. Int. 138, 447 (1999).

[27] P. A. Rydelek and I. S. Sacks, Geophys. Res. Lett. 28, 3079 (2001).

[28] T. Utsu, Y. Ogata, and S. Matsu'ura, J. Phys. Earth 43, 1 (1995).

[29] J. Dieterich, J. Geophys. Res. 99, 2601 (1994).
[30] A. Helmstetter, G. Ouillon, and D. Sornette, J. Geophys. Res. 108, 2483 (2003).

[31] A. Nur and J. R. Booker, Science 175, 885 (1972).

[32] J. Noir, E. Jacques, S. Bekri, P. M. Adler, P. Tapponnier, and G. C. P. King, Geophys. Res. Lett. 24, 2335 (1997).

[33] R. S. Stein, Nature (London) 402, 605 (1999).

[34] S. Toda, R. S. Stein, and T. Sagiya, Nature (London) 419, 58 (2002).

[35] T. Ouchi and T. Uekawa, Phys. Earth Planet. Inter. 44, 211 (1986).

[36] D. Marsan, C. J. Bean, S. Steacy, and J. McCloskey, J. Geophys. Res. 105, 28,081-28,094 (2000).

[37] A. Helmstetter and D. Sornette, Phys. Rev. E 66, 061104 (2002).

[38] K. R. Felzer and E. E. Brodsky, Nature (London) 441, 735 (2006).

[39] I. Main, Nature 441, 704 (2006).

[40] G. Rogers and H. Dragert, Science 300, 1942 (2003).

[41] Y. Ito, K. Obara, K. Shiomi, S. Sekine, and H. Hirose, Science 315, 503 (2007).

[42] R. M. Nadeau and D. Dolenc, Science 307, 389 (2005).

[43] S. Ide, G. C. Beroza, D. R. Shelly, and T. Uchide, Nature (London) 447, 76 (2007).

[44] H. Houston and J. E. Vidale, Nature 447, 49 (2007).

[45] I. A. Garagash, Dokl. Akad. Nauk 347, 95 (1996).

[46] D. N. Mikhailov and V. N. Nikolaevskiy, Izv., Phys. Solid Earth 36, 895 (2000).

[47] A. V. Vikulin, in Earthquake Source Asymmetry, Structural Media and Rotation Effects, edited by R. Teisseyre, M. Takeo, and E. Majewski (Springer, Heidelberg, 2006), pp. 273-289.

[48] V. G. Bykov, Izv., Phys. Solid Earth 37, 484 (2001).

[49] V. G. Bykov, in Earthquake Source Asymmetry, Structural Media, and Rotation Effects, edited by R. Teisseyre, M. Takeo, and E. Majewski (Springer, Heidelberg, 2006), pp. 241-253.

[50] S. R. Brown and C. H. Scholz, J. Geophys. Res. 90, 5531 (1985).

[51] R. Burridge and L. Knopoff, Bull. Seismol. Soc. Am. 57, 341 (1967)

[52] J. H. Dieterich, J. Geophys. Res. 77, 3771 (1972).

[53] J. B. Rundle and D. D. Jackson, Bull. Seismol. Soc. Am. 67, 1363 (1977).

[54] A. Nur, Pure Appl. Geophys. 116, 964 (1978).

[55] T. Cao and K. Aki, Pure Appl. Geophys. 122, 10 (1985).

[56] S. Brown, J. B. Rundle, and C. H. Scholz, Geophys. Res. Lett. 18, 215 (1991).

[57] J. M. Carlson, J. S. Langer, B. E. Shaw, and C. Tang, Phys. Rev. A 44, 884 (1991).

[58] K. Christensen and Z. Olami, J. Geophys. Res. 97, 8729 (1992).

[59] L. D. Landau and E. M. Lifshitz, Theory of Elasticity (Pergamon Press, New York, 1986).

[60] M. G. Forest and D. W. McLaughlin, Stud. Appl. Math. 68, 11 (1983).

[61] A. V. Gurevich, N. I. Gershenzon, A. L. Krylov, and N. G. Mazur, Dokl. Akad. Nauk. 34, 246 (1989).

[62] N. I. Gershenzon, Phys. Rev. B 50, 13308 (1994).

[63] J. C. Savage, J. Geophys. Res. 111, B04405 (2006).

[64] C. H. Scholz, The Mechanics of Earthquakes and Faulting (Cambridge University Press, Cambridge, 1990). 
[65] R. J. Archuleta, J. Geophys. Res. 89, 4559 (1984).

[66] O. M. Braun and Y. S. Kivshar, Phys. Rev. B 43, 1060 (1991).

[67] J. Weertman, Phys. Rev. Lett. 93, 205505 (2004).

[68] J. Lothe, Phys. Status Solidi B 242, 1978 (2005).
[69] D. H. Oppenheimer, Geophys. Res. Lett. 17, 1199 (1990).

[70] A. Michael, W. L. Ellsworth, and D. H. Oppenheimer, Geophys. Res. Lett. 17, 1441 (1990).

[71] G. C. Beroza and M. D. Zoback, Science 259, 210 (1993). 